\title{
ON THE COMPLETENESS OF THE PAPKOVICH POTENTIALS*
}

\author{
BY \\ ROBERT F. MILLAR \\ University of Alberta
}

\begin{abstract}
The Papkovich representation for the elastostatic displacement vector in a domain $D$ is considered. The possibility of eliminating from this representation either the scalar potential $\chi$ or a rectangular component $\psi$ of the vector potential $\psi$ is examined. Earlier work is discussed and the connection is made with the oblique derivative problem of potential theory. A convexity requirement on the boundary of $D$ is shown to be necessary in general in order that $\chi$ or $\psi$ may be eliminated.. A result of Stippes for a domain with an internal cavity is generalized, and two new classes of domains are found for which $\chi$ may be eliminated.
\end{abstract}

1. Introduction. In a linear, homogenous, isotropic elastic medium that occupies a domain $D$ of $E^{3}$, the displacement vector $\mathbf{u}$ satisfies the equation

$$
\Delta \mathbf{u}+(1-2 v)^{-1} \nabla \nabla \cdot \mathbf{u}=\mathbf{0} .
$$

Here $\nu\left(-1<\nu<\frac{1}{2}\right)$ denotes Poisson's ratio, and body forces are assumed to be absent.

A general solution to (1.1) may be written in the form

$$
\mathbf{u}=\nabla(\chi+\mathbf{r} \cdot \psi)-\alpha \psi, \quad \Delta \chi=0, \Delta \psi=\mathbf{0},
$$

in $D$, wherein $r$ is the position vector with respect to a given origin, and $\alpha:=4(1-\nu)$ so $2<\alpha<8$. The functions $\chi$ and $\psi$ are called the Papkovich potentials. It is always assumed without further comment that any conditions on $\mathbf{u}$ or $\partial D$ that are necessary for the validity of (1.2) are fulfilled; see, for example, [1, 2, p. 329, 3, p. 141]. Moreover, if $D$ is unbounded, it is assumed that

$$
\chi=O\left(r^{-1}\right), \quad|\nabla \chi|=O\left(r^{-2}\right), \quad \text { as } r \rightarrow \infty,
$$

and similarly for $\psi$; thus $\chi$ and $\psi$ are harmonic whether $D$ is bounded or unbounded. This permits a more unified treatment, though in practice it may be somewhat restrictive.

\footnotetext{
* Received August, 31, 1981. Publication of this paper was inadvertently delayed. This work was done while the author was a guest of the Applied Mathematics Institute, Department of Mathematical Sciences, University of Delaware. The writer wishes to express his gratitude to the Institute and to the Department for hospitality and support. In particular, he thanks Dr. R. P. Gilbert, Director of the Institute, for bringing this problem to his attention.

A more detailed version of this work may be obtained from the author.
} 
Since the Papkovich potentials involve four scalar functions whereas $\mathbf{u}$ involves only three, it is natural to ask whether the number of harmonic functions can be reduced to three. It is this question that we shall address. The answer will turn out to be quite dependent on the choice of origin and the orientation of the coordinate system. Historical reviews of this problem have been given by Eubanks and Sternberg [1], and Gurtin [3, §44].

In the next section, we discuss briefly some relevant earlier work on the problem. The connection with the oblique derivative problem of potential theory, first noted by Stippes [4], is made in Sec. 3. The convexity requirement in [1] for the elimination of a component of $\psi$ is shown to be necessary in general, and an analogous requirement is inferred for the elimination of $\chi$ (Theorem 1). In Sec. 4, a result of Stippes [4] concerning the elimination of $\chi$ when $D$ is periphractic (that is, when $D$ is bounded and has an internal cavity) is generalized (Theorem 2). In Sec. 5, two new classes of domains for which $\chi$ may be taken to be zero are determined (Theorems 3, 4). Some final remarks are made in Sec. 6.

We conclude this section by defining two terms that occur frequently in the subsequent discussion.

A domain $D$ that does not contain the origin 0 will be said to be $r$-convex (or radially convex) if there exists a surface $\Sigma$ whose intersection with $D$ has the following properties:

(i) $S:=\Sigma \cap D$ is not empty.

(ii) $S$ is analytic.

(iii) $S$ is star-like with respect to 0 ; that is, $\hat{n} \cdot \hat{r} \neq 0$, where $\hat{n}$ is a unit normal to $S$ and $\hat{r}$ is a unit vector in the radial direction. Thus, in spherical polar coordinates, $S$ has a representation of the form $r=r_{0}(\theta, \phi)$ when $(\theta, \phi)$ ranges over some domain on the unit sphere.

(iv) The radial line through each point of $S$ intersects $D$ in an interval.

(v) Every point of $D$ lies on some radial line through $S$.

In the limiting form of the above, when $D$ remains fixed and the origin 0 moves to infinity in the $z$-direction, these properties characterize a $z$-convex domain. Here a "radial line" becomes a "line parallel to the $z$-axis", $\hat{r}$ is replaced by $\hat{z}$, and $S$ has a representation of the form $z-f(x, y)$ in some domain of the $x, y$-plane.

It is evident that the boundary of an $r$-convex domain may contain segments of radial lines, and the boundary of a $z$-convex domain may contain segments of lines that are parallel to the $z$-axis.

2. Some earlier results. The early history of this problem is reviewed in [1] and [3]. Sokolnikoff [2, pp. 328-334] showed that $\chi$ may be eliminated from (1.2) provided that it can be represented throughout $D$ by a series of solid harmonics. If $D$ is bounded, it is necessary to assume that $\alpha$ is not equal to an integer. When $D$ is bounded, Sokolnikoff's proof requires that $\chi$ be harmonic not only in $D$ but throughout some sphere containing $D$; and similarly when $D$ is unbounded, there must be a sphere in the complement of $D$ outside of which $\chi$ is harmonic. Furthermore, if $\partial D$ is a sphere, then a rectangular component of $\psi$ can be eliminated from (1.2). Eubanks and Sternberg [1] deduced that (i) $\chi$ may be replaced by zero without loss in completeness provided $D$ is star-shaped with respect to the origin and $\alpha$ is not an integer; (ii) if $D$ is $z$-convex, the $z$-component of $\psi$ 
may be eliminated without loss in completeness. Later, Stippes [4] extended the result (i) of [1] to the simplest periphractic domains containing a single cavity, and to unbounded domains, and he obtained the form of the representation when $\alpha$ is an integer. In the periphractic case it was assumed that a sphere lying wholly in $D$ and with center at the origin could be constructed; it will be shown later that this restriction is unnecessary. Cong and Steven [5] used elementary arguments to examine the possibility of eliminating $\chi$ from (1.2). For bounded $D$, they obtained the result (i) of [1] and, when $D$ was unbounded they found a result similar to one of Sokolnikoff [2] and to Theorem 5 of [4].

\section{Connection with the oblique derivative problem.}

3.1 Elimination of the scalar potential. The harmonic potential $\chi$ may be eliminated if and only if there exists a function $\Phi \in C^{2}(D)$ such that

$$
\Delta \Phi=0, \quad \mathbf{r} \cdot \nabla \Phi-\alpha \Phi=\chi,
$$

in $D$; see [1]-[4]. Although it will be characterized by the boundary conditions on $\partial D, \chi$ is unknown and we are obliged to assume that it is an arbitrary harmonic function in $D$.

It was Stippes [4] who first observed that (3.1) is a particular case of the oblique derivative problem. For this, a function $\Phi$ is sought that is harmonic in $D$ and that satisfies the boundary condition $\nu \cdot \nabla \Phi+a \Phi=b$, where $a$ and $b$ are sufficiently smooth functions and $\nu(\neq 0)$ is a sufficiently smooth direction field on $\partial D$. The problem is said to be regular if $\nu$ is nowhere tangent to $\partial D$; if $\nu$ is tangent to $\partial D$ at any point, the problem is non-regular.

In two dimensions, the oblique derivative problem has been solved completely; see, for example [6, Chapter III], [7, Chapter VI], [8, Chapter 9]. For $n(>2)$ dimensions, a complete treatment of the regular problem was given some time ago by reducing it to a singular integral equation to which, none the less, the Fredholm alternative applies; for example, see [7, Chapter V, §4], [9, §43], [10, §23]. Although rather specific yet illuminating examples had been considered previously [7, Chapter VII], it was only with the appearance in 1966 of a paper by Hörmander [11] that a satisfactory picture of the non-regular case began to emerge; see Winzell [12] for recent references. In this case, the Fredholm alternative does not apply, a solution may or may not exist and, if it does exist it will not be unique unless additional conditions are imposed. Hörmander [11] gave both existence and non-existence results, but little or no attention appears to have been directed subsequently to the case for which existence of a solution is not guaranteed. This, unfortunately, is a case that is of special interest to us.

For the problem under consideration at present, $\Phi$ must satisfy (3.1) throughout $D$. We shall assume that the second of (3.1) holds also on $\partial D$. But, given that $\chi$ and $\Phi$ are harmonic in $D$, by considering the harmonic function $\mathbf{r} \cdot \nabla \Phi-\alpha \Phi-\chi$ it is easy to see that if this condition holds on $\partial D$ then it holds automatically throughout $D$. Thus the problem

$$
\Delta \Phi=0 \quad \text { in } D, \quad \mathbf{r} \cdot \nabla \Phi-\alpha \Phi=\chi \quad \text { on } \partial D,
$$

is equivalent to (3.1), and is that of the oblique derivative with the direction field $\nu \equiv \mathbf{r}=(x, y, z)$. 
If $D$ is bounded, the significance of the star-shaped requirement in the results described in Sec. 2 is now apparent: the oblique derivative problem is regular, the Fredholm alternative applies and, if $\alpha$ is not a positive integer $(2<\alpha<8)$, (3.2) has a unique solution; thus $\chi$ can be eliminated from (1.2). If $\alpha$ is a positive integer, then (3.2) has a solution if and only if $\chi$ is orthogonal on $\partial D$ to an arbitrary solid harmonic of degree $\alpha$; this is an eigenfunction of a certain integral operator. When this condition is fulfilled, $\chi$ may be eliminated from (1.2) but, in general, only the component of $\chi$ that is orthogonal to the solid harmonic can be omitted; see [4, Theorem 4]. If the complement of $D \cup \partial D$ is bounded and star-shaped with respect to the origin, then the Fredholm alternative again applies [4, Theorem 5]. In this case, $\chi$ can be eliminated for all $\alpha$.

If $D$ is not star-shaped with respect to 0 , then the problem is non-regular. We shall discuss this later. But first we shall consider the possibility of eliminating a component of $\psi$.

3.2 Elimination of a component of $\psi$. The $z$-component of $\psi(\psi$, say) can be eliminated from (1.2) if and only if there exists a function $H \in C^{2}(D)$ such that

$$
\Delta H=0, \quad H_{z}=\psi,
$$

in $D$, where $\psi$ is harmonic; see $[1,2$, p. 333,3, p. 146]. Both $\psi$ and $H$ are analytic in $D$. This problem is equivalent to an oblique derivative problem for $H$ in $D$, with $\boldsymbol{\nu}$ parallel to the $z$-axis. If $D$ is bounded and $z$-convex, and $\partial D$ is smooth, then the problem necessarily is non-regular; it may or may not be non-regular if $D$ is unbounded and $z$-convex. When $D$ is bounded and $z$-convex, existence of a solution may be inferred from [11, p. 202] and an explicit solution can be found [1, p. 743]; similar examples have been discussed in [7, p. 146, pp. 196-198]. In these circumstances, Eubanks and Sternberg [1] concluded that $\psi$ may be eliminated from (1.2).

If property (iv) in the definition of $z$-convexity is violated, while all remaining conditions are satisfied, then (3.3) may or may not have a solution throughout $D$. We shall assume that some lines parallel to the $z$-axis intersect $D$ in two intervals, and that these intervals fill positive volume. Thus $\partial D$ will have a concave or re-entrant portion $B$, say, and the possibility of non-existence of a solution for arbitrary $\psi$ may be inferred from [11, p. 202]. In Hörmander's notation, at a point of $B$ where a line parallel to the $z$-axis is tangent, the geometry corresponds to $\nu_{3}=0, \Sigma_{1}^{2} \nu_{j} \partial \nu_{3} / \partial x_{j}>0$; the sign of the outward normal component of $\boldsymbol{\nu}$ on $\partial D$ changes from positive to negative when following $\boldsymbol{\nu}$ along $\partial D$ in the direction induced by the tangential component of $\nu$ on $\partial D$. That a solution may not exist even when $\psi$ is harmonic can be shown explicitly as follows.

Through each point of the analytic surface $S$ we draw a line segment that is parallel to the $z$-axis, the interior of which consists entirely of points of $D$, and whose endpoints lie on $\partial D$. Denote by $D^{\prime}$ the union of all such line segments. Then $D^{\prime}$ is connected, $z$-convex, and $D^{\prime} \subset D$. By hypothesis, $S$ has a representation of the form $z=f(x, y),(x, y) \in S^{\prime}$, where $S^{\prime}$ is the projection of $S$ onto the $x, y$-plane, and $f$ is analytic. For simplicity, we assume that $S^{\prime}$ is bounded. 
A solution to (3.3) in $D^{\prime}$ may be constructed as in [1] or [3, p. 146]:

$$
\begin{aligned}
H(x, y, z)= & \int_{f(x, y)}^{z} \psi(x, y, \eta) d \eta \\
& +(2 \pi)^{-1} \int_{S^{\prime}} F(\xi, \eta) \log R d \xi d \eta+\lambda(x, y) .
\end{aligned}
$$

Here $R^{2}:=(x-\xi)^{2}+(y-\eta)^{2}, \quad F(x, y):=\psi \Delta_{2} f+2\left(\psi_{x} f_{x}+\psi_{y} f_{y}\right)+\psi_{z}\left(f_{x}^{2}+f_{y}^{2}-\right.$ 1), $\Delta_{2} \lambda=0, \Delta_{2}$ is the two-dimensional Laplacian, and $\lambda$ is analytic in $S^{\prime}$. That the integral involving $F$ is analytic in $S^{\prime}$ may be shown by adapting Levi's method [13, Chapter II, §6] to this two-dimensional situation. It is apparent that $H$ is determined only to within an arbitrary harmonic function in $S^{\prime}$.

The part of $\partial D^{\prime}$ that lies in $D$ is generated by segments of lines parallel to the $z$-axis that are tangent to $\partial D$. (It is assumed that $\partial D$ is smooth.) In general, it is not possible to extend the solution $H$ analytically across this part of $\partial D^{\prime}$. For let $l$ denote one of the line segments that generates this portion of $\partial D^{\prime}$, and choose the origin $0 \notin D$ so that $l$ is part of the line $x=0, y=\varepsilon$ (where $\varepsilon$ is positive but small), and that $l$ is tangent to $\partial D$ at $(0, \varepsilon, 0)$. Let

$$
\psi(x, y, z):=\left(x^{2}+y^{2}+z^{2}\right)^{-1 / 2} .
$$

Since $0 \notin D, \psi$ is harmonic in $D$. The first integral in (3.4) may be evaluated for $(x, y, z) \in D^{\prime}$, and is analytic. But if $\varepsilon \downarrow 0$, so that the singularity of $\psi$ approaches the point at which $l$ is tangent to $\partial D$, the integral becomes logarithmically infinite everywhere on the part of $\partial D^{\prime}$ that has $l$ for generator. Consequently if $\psi$ is given by (3.5), there is no solution to (3.3) throughout $D$.

It is also clear that a singularity of $\psi$ at the above origin does not necessarily induce a singularity of $H$ in $D$. For example, we need only take for $\psi$ in (3.3) the $z$-derivative of $\psi$ in (3.5). In fact, from (3.3) it is obvious that a solution exists if and only if $\psi$ is the $z$-derivative of a harmonic function in $D$.

If $D$ is not radially convex in the sense that property (iv) is not fulfilled, Hörmander's result shows that it may not be possible to eliminate $\chi$ from (1.2). An explicit example on the lines of that discussed above can also be constructed.

These results are summarized in the following theorem.

THEOREM 1. If $D$ is not $z$-convex in the sense that property (iv) does not hold, then in general the $z$-component of $\psi$ cannot be eliminated from (1.2). If $D$ is not $r$-convex in the sense that property (iv) does not hold, then in general the potential $\chi$ cannot be eliminated from (1.2).

4. Generalization of a result of Stippes. In [4], Stippes investigated the problem of eliminating $\chi$ from (1.2) by formulating it in terms of a singular integral equation. The volume under consideration was bounded by two closed surfaces $S_{0}, S_{1}$. The origin 0 was inside $S_{0}$ which was, in turn, inside $S_{1}$. Each surface was star-like with respect to 0 . In the present section, we show that Stippes' Theorem 6 can be strengthened. For ease of comparison, we shall retain most of the notation of [4]. 
Stippes' Theorem 6 concerns a solution to (3.1) in the domain $D$ between $S_{0}$ and $S_{1}$. The star-like requirement implies the validity of the Fredholm alternative $[9, \S 43,10, \S 23]$, so existence of a solution $\Phi$ follows if $\chi$ is orthogonal to all solutions to a certain homogenous singular integral equation on $\partial D$. Let $\beta$ denote any solution to this equation. Following standard procedure, Stippes introduced the single layer potential $\Psi$, with density $\beta$. If $D_{0}^{*}$ denotes the domain interior to $S_{0}$ and $D_{1}^{*}$ is the domain exterior to $S_{1}$, let $\Psi^{-}(P):=\Psi(P), P \in D_{0}^{*} \cup D_{1}^{*}$ and $\Psi^{+}(P):=\Psi(P), P \in D$. Then the orthogonality condition [4, Eq. 18] may be written in terms of outer normal derivatives of $\Psi^{+}, \Psi^{-}$as

$$
\int_{\partial D} \chi\left(\partial \Psi^{+} / \partial n-\partial \Psi^{-} / \partial n\right) d Q=0
$$

When $\alpha$ is not a positive integer, it may be shown that $\Psi^{-}(P) \equiv 0, \Psi^{+}(P) \equiv 0$; thus (4.1) is satisfied and $\chi$ may be eliminated from (1.2).

If $\alpha$ is a positive integer, the situation is less simple. We shall modify Stippes' arguments $[4, \S 4]$ to yield a result stronger than his Theorem 6 . In this case, by permitting $P$ to move onto $\partial D$, and by recalling the definition of $\beta$, it may be shown that $\Psi^{-}=0$ on $S_{0}$ and $\Psi^{-}=H_{-\gamma}$ on $S_{1}$, where $\gamma:=\alpha+1$ and $H_{-\gamma}$ is an arbitrary solid harmonic of order $-\gamma$ (radial dependence $r^{-\gamma}$ ). Thus $\Psi^{-}=0$ in $D_{0}^{*}, \Psi^{-}=H_{-\gamma}$ in $D_{1}^{*}$. From the continuity properties of single layer potentials, it follows that $\Psi^{+}=0$ on $S_{0}$ and $\Psi^{+}=H_{-\gamma}$ on $S_{1}$. These results, together with the use of Green's third identity in $D$, permit (4.1) to be written as

$$
\int_{S^{\prime}}\left(H_{-\gamma} \partial \chi / \partial n-\chi \partial H_{-\gamma} / \partial n\right) d Q=0,
$$

where $S^{\prime}$ is any smooth, closed surface between $S_{0}$ and $S_{1}$.

In general, (4.2) is not satisfied. But, by writing $\chi^{\prime}:=\chi-H_{\alpha}$ where $\chi^{\prime}$ is the component of $\chi$ that is orthogonal to the solid harmonic $H_{\alpha}$ on $\partial D$, and by assuming that $S^{\prime}$ could be a sphere centered on 0 , Stippes was able to argue that $\chi^{\prime}$ could be eliminated. That this condition on $S^{\prime}$ is unnecessary may be seen as follows.

At any point $P$ in $D$ the harmonic function $\chi$ in (1.2) may be represented by an integral involving the fundamental solution and the values of $\chi$ and $\partial \chi / \partial n$ on $S_{0} \cup S_{1}$. If $\chi_{i}$ $(i=0,1)$ denotes the function determined by the integral on $S_{i}$, then $\chi(P)=\chi_{0}(P)+$ $\chi_{1}(P), P \in D$. Here $\chi_{0}$ is harmonic outside $S_{0}$ (and is zero at infinity), while $\chi_{1}$ is harmonic inside $S_{1}$. Thus, if $P_{\alpha}(\chi, \psi)$ denotes the right-hand side of (1.2),

$$
\mathbf{u}=P_{\alpha}\left(\chi_{0}, \psi\right)+P_{\alpha}\left(\chi_{1}, \psi\right) \text {. }
$$

We show that $\chi_{0}$ can be eliminated. This follows if (4.2) is satisfied with $\chi$ replaced by $\chi_{0}$. Because both $\chi_{0}$ and $H_{-\gamma}$ are harmonic outside $S_{0}, S^{\prime}$ may be expanded into a sphere whose radius tends to infinity. Since $\gamma>0$ and $\chi_{0}$ is zero at infinity, the integral is zero and $\chi_{0}$ can be eliminated from $P_{\alpha}\left(\chi_{0}, \psi\right)$ to give $P_{\alpha}\left(\chi_{0}, \psi\right)=P_{\alpha}\left(0, \psi^{\prime}\right)$ for a suitable $\psi^{\prime}$.

In general, $\chi_{1}$ cannot be eliminated from $P_{\alpha}\left(\chi_{1}, \psi\right)$ since (4.2) with $\chi$ replaced by $\chi_{1}$ will not usually be satisfied. In fact, $S^{\prime}$ here may be drawn down onto a small sphere centered on 0 . Moreover, inside and on this sphere $\chi_{1}$ may be expanded in a series of solid harmonics: $\chi_{1}(P)=\sum_{0}^{\infty} H_{n}$, and the integral (4.2) vanishes only if $\chi_{1}$ is independent of $H_{\alpha}$. Thus we are led to define $\chi_{1}^{\prime}$ by $\chi_{1}^{\prime}:=\chi_{1}-H_{\alpha}$. Then $\chi_{1}^{\prime}$ satisfies the orthogonality 
condition, leading to $P_{\alpha}\left(\chi_{1}, \psi\right)=\nabla H_{\alpha}+P_{\alpha}\left(0, \psi^{\prime \prime}\right)$. Consequently (4.3) becomes $\mathbf{u}=\nabla H_{\alpha}$ $+P_{\alpha}\left(0, \psi^{\prime}+\psi^{\prime \prime}\right)$, or $\mathbf{u}=P_{\alpha}\left(H_{\alpha}, \psi\right)$, where now $\psi:=\psi^{\prime}+\psi^{\prime \prime}$. We have the following theorem.

THEOREM 2 (STIPPES, GENERALIZED). If $D$ is the periphractic domain bounded internally by $S_{0}$ and externally by $S_{1}$, and if $S_{0}$ and $S_{1}$ are star-like with respect to 0 , then $P_{\alpha}(0, \psi)$ is complete in $D$ when $\alpha$ is not a positive integer $(2<\alpha<8)$. If $\alpha$ is a positive integer and $H_{\alpha}$ is an arbitrary solid harmonic of order $\alpha$, then $P_{\alpha}\left(H_{\alpha}, \psi\right)$ is complete in $D$.

5. New domains for which $\chi$ may be eliminated. None of the recent work on the oblique derivative problem, except for that of Hörmander [11] and Bitsadze [7], is particularly useful in deducing a domain for which $\chi$ may be eliminated. We shall show directly that this can be done in two new fairly general cases. We suppose first that $D$ is bounded and then unbounded domains will be considered.

In all previous studies, the origin 0 has been chosen to give a regular problem. Here it is assumed that $0 \notin D$ and that it is possible to choose the polar axis $\theta=0$ in such a way that $D$ lies inside a circular cone with vertex 0 and half-angle $\gamma$ satisfying $0<\gamma<\pi$. Since $D$ is bounded the problem is non-regular if $\partial D$ is smooth. It will be assumed that $D$ is $r$-convex. Then the analytic surface $S$ may be represented in spherical polar coordinates by an equation of the form $r=r_{0}(\theta, \phi),(\theta, \phi) \in S^{\prime}$, wherein $r_{0}$ is an analytic function with domain $S^{\prime}$ in $0 \leqslant \theta<\gamma, 0 \leqslant \phi \leqslant 2 \pi$, on the unit sphere.

We now show that a solution to (3.1) can be constructed in a manner similar to that of [1] or [5]. The second of (3.1) is integrated to give

$$
\Phi(r, \theta, \phi)=r^{\alpha} \Psi(\theta, \phi)+r^{\alpha} \int_{r_{0}}^{r} \eta^{-\alpha-1} \chi(\eta, \theta, \phi) d \eta .
$$

Here $r_{0}:=r_{0}(\theta, \phi)$ and $\Psi(\theta, \phi):=r_{0}^{-\alpha} \Phi\left(r_{0}, \theta, \phi\right) ; \Psi$ is analytic. By writing $\Delta$ as $\Delta_{r}+$ $r^{-2} \Delta_{\theta \phi}$, where $\Delta_{r}$ is a radial operator and $\Delta_{\theta \phi}$ is the Laplace-Beltrami operator on the unit sphere, it is straight forward to show that $\Delta \Phi=r^{\alpha-2}\left[\Delta_{\theta \phi} \Psi+\alpha(\alpha+1) \Psi-\Lambda\right]$; here $\Lambda$ depends on $\chi, r_{0}$, and their derivatives, and is analytic for $(\theta, \phi) \in S^{\prime}$. It follows that $\Phi$ is harmonic in $D$ if and only if

$$
\Delta_{\theta \phi} \Psi+\alpha(\alpha+1) \Psi=\Lambda, \quad(\theta, \phi) \in S^{\prime} .
$$

For our purposes, any particular solution $\Psi_{P}$ to (5.2) will suffice. Such a solution may be obtained by using a fundamental solution for a closely related equation, given by Vekua [6, §14]. Following Vekua, we make the change of variables $x=\tan \frac{1}{2} \theta \cos \phi$, $y=\tan \frac{1}{2} \theta \sin \phi$. If $\Psi(\theta, \phi) \rightarrow U(x, y)$ and $\Lambda(\theta, \phi) \rightarrow h(x, y)$, it is found that

$$
\Delta_{2} U+4 \alpha(\alpha+1)\left(1+x^{2}+y^{2}\right)^{-2} U=F,
$$

wherein $F(x, y):=4\left(1+x^{2}+y^{2}\right)^{-2} h(x, y)$, and $F$ is analytic.

This coordinate transformation represents the stereographic projection from the south pole of a point on the unit sphere onto the $x, y$-plane. Under the transformation, a particular solution to (5.3) becomes a particular solution to (5.2). Since $D$ does not include $\theta=\pi, S^{\prime}$ will transform into a bounded domain $S^{\prime \prime}$ in the $x, y$-plane. 
Vekua's fundamental solution is

$$
\omega(x, y ; \xi, \eta):=(2 \pi)^{-1}\left\{-P_{\alpha}(X) \log R+\int_{0}^{1} \log t \frac{\partial}{\partial t} P_{\alpha}[X+(1-X) t] d t\right\},
$$

in which $X:=\left(1-R^{2}\right) /\left(1+R^{2}\right), R^{2}:=(x-\xi)^{2}+(y-\eta)^{2}$, and in which $P_{\alpha}$ denotes the Legendre function of the first kind of index $\alpha$. It is apparent that $\omega$ depends only on $R$. Moreover, $-1+2 /\left(1+R^{2}\right) \leqslant X+(1-X) t \leqslant 1$ when $0 \leqslant t \leqslant 1$, so $\omega$ is defined for all finite positive values of $R$. Then, since $P_{\alpha}(1)=1$, a particular solution to (5.3) is

$$
U_{P}(x, y):=-\int_{S^{\prime \prime}} F(\xi, \eta) \omega(x, y ; \xi, \eta) d \xi d \eta,
$$

from which $\Psi_{P}$ may be found. Analyticity of $U_{P}$ (and, hence, of $\Psi_{P}$ ) may be shown by using Levi's method [13, Chapter II, §6].

It follows that (3.1) has a solution and, consequently, that $\chi$ may be eliminated from (1.2). We sum this up as a theorem.

TheOREM 3. Let $D$ be a bounded, radially convex domain that lies inside a circular cone $\theta=\gamma$, with vertex 0 and with $0<\gamma<\pi$. Then the scalar potential $\chi$ may be eliminated from (1.2).

The boundedness requirement can be lightened, and $D$ can extend to infinity, if $D$ is radially convex and $r_{0}$ is bounded on $S^{\prime} \cup \partial S^{\prime}$. For example, this is the situation when $D$ is the interior of a paraboloid of revolution about the $z$-axis.

If $r_{0}(\theta, \phi)$, or a derivative, becomes unbounded on approach to $\partial S^{\prime}$ (for example, if $D$ is the interior of one sheet of a hyperboloid of revolution), it is not clear that the integral in (5.4) is convergent. If it is assumed that a radial line cuts $\partial D$ transversely in at most one point then, since $\chi$ satisfies (1.3) and $\alpha>2,(5.1)$ may be replaced by

$$
\Phi(r, \theta, \phi)=-r^{\alpha} \int_{r}^{\infty} \eta^{-\alpha-1} \chi(\eta, \theta, \phi) d \eta .
$$

Then $\Phi$ fulfils all requirements and $\chi$ may be eliminated from (1.2). Consequently we have the following theorem.

TheORem 4. Let $D$ be an unbounded domain not containing 0 , but contained in a cone with vertex 0 and half-angle less than $\pi$. Suppose that a radial line from the origin cuts $\partial D$ transversely in at most one point. Then $\chi$ may be eliminated from (1.2).

It is noted that the case in which the complement of $D$ is bounded and star-shaped with respect to 0 has been considered in [4] and [5].

6. Concluding remarks. We summarize the present state regarding the elimination of (a) the $z$-component of $\psi$ or (b) $\chi$ from (1.2), subject to any necessary smoothness conditions or conditions at infinity.

(a) If $D$ is $z$-convex, then the $z$-component of $\psi$ can be eliminated.

(b) $\chi$ can be eliminated in the following cases:

(i) if $0 \in D$, if $D$ is bounded and star-shaped with respect to 0 , and if $\alpha$ is not an integer $(2<\alpha<8)$. 
(ii) if $D$ is bounded and $r$-convex.

(iii) if $D$ is unbounded and a radial line cuts $\partial D$ transversely in at most one point.

(iv) if $D$ is the bounded, periphractic domain between two surfaces that are star-like with respect to 0 .

In case (i) of (b), if $\alpha$ is an integer then $\chi$ can be eliminated only to within a term $H_{\alpha}$.

There remains the question of the case in which $\partial D$ is re-entrant with respect to the $z$ - or $r$-direction and convexity property (iv) does not hold. Hörmander's result [11, p. 202], or the example of Sec. 3, shows that a solution to (3.3), or to (3.1), may or may not exist throughout $D$. The resolution of this question appears to be a difficult task.

\section{REFERENCES}

[1] R. A. Eubanks and E. Sternberg, On the completeness of the Boussinesq-Papkovich stress functions, J. Rat. Mech. and Anal. 5, 735-746 (1956)

[2] I. S. Sokolnikoff, Mathematical theory of elasticity, second edition, McGraw-Hill Book Company, New York, 1956

[3] M. E. Gurtin, The linear theory of elasticity, Encyclopedia of Physics, Volume VIa/2, Springer-Verlag, Berlin, Heidelberg, New York, 1972, pp. 1-295

[4] M. Stippes, Completeness of the Papkovich potentials, Quart. Appl. Math. 26, 477-483 (1969)

[5] T. Tran Cong and G. P. Steven, On the representation of elastic displacement fields in terms of three harmonic functions, J.Elasticity 9, 325-333 (1979)

[6] I. N. Vekua, New methods for solving elliptic equations, North-Holland Publishing Company, Amsterdam, 1967

[7] A. V. Bitsadze, Boundary value problems for second order elliptic equations, North-Holland Publishing Company, Amsterdam, 1968

[8] N. I. Muskhelishvili, Singular integral equations, P. Noordhoff Ltd., Groningen, 1953

[9] S. G. Mikhlin, Multidimensional singular integrals and integral equations, Pergamon Press, Oxford, 1965

[10] C. Miranda, Partial differential equations of elliptic type, second revised edition, Springer-Verlag, Berlin, 1970

[11] L. Hörmander, Pseudo-differential operators and non-elliptic boundary problems, Ann. of Math. 83, 129-209 (1966)

[12] B. Winzell, $A$ boundary value problem with an oblique derivative, Comm. Partial Differential Equations 6 , 305-328 (1981)

[13] S. Bochner and W. T. Martin, Several complex variables, Princeton University Press, Princeton, 1948 